\title{
ON THE STRUCTURE OF SKEW SYMMETRIC OPERATORS
}

\begin{abstract}
SEN ZHU
Abstract. An operator $T$ on a complex Hilbert space $\mathscr{H}$ is called skew symmetric if $T$ can be represented as a skew symmetric matrix relative to some orthonormal basis for $\mathscr{H}$. We use multiplicity theory to characterize when there is an anti-conjugation commuting with a fixed positive operator, and give a description of such anti-conjugations. Based on these results, we provide a canonical model of skew symmetric operators in terms of multiplication operators on function spaces.
\end{abstract}

Mathematics subject classification (2010): Primary 47A45, 47B99; Secondary 47A68.

Keywords and phrases: Skew symmetric operator, complex symmetric operator, polar decomposition, multiplication operator.

\section{REFERENCES}

[1] N. Chevrot, E. Fricain, And D. Timotin, The characteristic function of a complex symmetric contraction, Proc. Amer. Math. Soc. 135 (2007), no. 9, 2877-2886 (electronic).

[2] J. B. Conway, A course in functional analysis, second ed., Graduate Texts in Mathematics, vol. 96, Springer-Verlag, New York, 1990.

[3] S. R. GARCIA AND D. E. POORE, On the norm closure of the complex symmetric operators: compact operators and weighted shifts, J. Funct. Anal. 264 (2013), no. 3, 691-712.

[4] S. R. Garcia And M. Putinar, Complex symmetric operators and applications, Trans. Amer. Math. Soc. 358 (2006), no. 3, 1285-1315 (electronic).

[5] S. R. Garcia And M. Putinar, Complex symmetric operators and applications, II, Trans. Amer. Math. Soc. 359 (2007), no. 8, 3913-3931 (electronic).

[6] S. R. Garcia, J. E. Tener, Unitary equivalence of a matrix to its transpose, J. Operator Theory 68(2012), no. 1, 179-203.

[7] S. R. Garcia And W. R. Wogen, Complex symmetric partial isometries, J. Funct. Anal. 257 (2009), no. $4,1251-1260$.

[8] T. M. Gilbreath And W. R. Wogen, Remarks on the structure of complex symmetric operators, Integral Equations Operator Theory $\mathbf{5 9}$ (2007), no. 4, 585-590.

[9] K. GUO, Y. Ji, AND S. ZHU, A $C^{*}$-algebra approach to complex symmetric operators, Trans. Amer. Math. Soc. 367 (2015), no. 10, 6903-6942.

[10] K. GUO AND S. ZHU, A canonical decomposition of complex symmetric operators, J. Operator Theory 72 (2014), no. 2, 529-547.

[11] R. A. Horn And C. R. Johnson, Matrix analysis, Corrected reprint of the 1985 original, Cambridge University Press, Cambridge, 1990.

[12] C. G. Li And T. T. Zhou, Skew symmetry of a class of operators, Banach J. Math. Anal. 8 (2014), no. $1,279-294$.

[13] C. G. Li And S. ZhU, Skew symmetric normal operators, Proc. Amer. Math. Soc. 141 (2013), no. 8, $2755-2762$.

[14] S. M. ZAGORODNYUK, On a J-polar decomposition of a bounded operator and matrices of $J$ symmetric and J-skew-symmetric operators, Banach J. Math. Anal. 4 (2010), no. 2, 11-36.

[15] S. M. ZAGORODNYUK, On the complex symmetric and skew-symmetric operators with a simple spectrum, Symmetry, Integrability and Geometry: Methods and Applications 7 (2011), 1-9.

[16] S. ZHU, Approximate unitary equivalence to skew symmetric operators, Complex Anal. Oper. Theory 8 (2014), no. 7, 1565-1580. 
[17] S. ZHU, Skew symmetric weighted shifts, Banach J. Math. Anal. 9 (2015), no. 1, 253-272.

[18] S. ZHU AND C. G. LI, Complex symmetry of a dense class of operators, Integral Equations Operator Theory 73 (2012), no. 2, 255-272.

[19] S. ZHU And C. G. Li, Complex symmetric weighted shifts, Trans. Amer. Math. Soc. 365 (2013), no. $1,511-530$.

[20] S. Zhu And J. Zhao, The Riesz decomposition theorem for skew symmetric operators, J. Korean Math. Soc. 52 (2015), no. 2, 403-416. 